\title{
Exploration of an Open Online Learning Platform Based on Google Cloud Computing
}

\author{
https://doi.org/10.3991/ijet.v12i07.7249 \\ Kai Zhao* \\ North China University of Water Resources and Electric Power, Zhengzhou, China \\ HenNan Agricultural University, Zhengzhou, China \\ zhaozhangk@126.com \\ Qingxue Yang \\ North China University of Water Resources and Electric Power, Zhengzhou, China \\ Xinming Ma \\ HenNan Agricultural University, Zhengzhou, China
}

\begin{abstract}
With the progress of society, traditional online learning platforms have demonstrated the uneven distribution of information resources, and teacher-student communication exhibits a certain delay. At present, cloud computing, which is a new product of information technology, has been favored in many areas because of its superior feedback mechanism and storage space. Therefore, to improve the integration of online learning information resources and facilitate interaction between teachers and students, we designed our own online learning system based on the Google cloud computing platform. We used Google's cloud computing platform and the Google App Engine to develop a unified and open online learning platform that is capable of storing large amounts of data, integrating considerable amounts of learning resources, and storing them on cloud. Through a test, we determined that the designed online learning platform for sharing information resources and integrating teacherstudent exchanges is highly beneficial. The platform helps the classroom learning atmosphere become active, and has a positive effect on teaching methods. The proposed platform can promote further development of online learning.
\end{abstract}

Keywords-Google Cloud Computing; open; online learning

\section{Introduction}

With the advent of the Information Age, online learning, as a new aspect of modern education, conforms to the development of the current knowledge-based society, thereby allowing people to create a ubiquitous learning space that does not only increase the flexibility of learning arrangements, but also achieve the need for lifelong education [1]. However, after years of full-swing development, the following issues remain in the current online learning system in China: low utilization of learning resource developments and poor degree of sharing. The lack of cooperation among 
universities or distance education institutions, as well as their independent integration of developments, results in the information island phenomenon. A large number of resources cannot be shared, thereby repeating production and wasting human and material resources. The online learning system is the support platform for online learning. However, current educational institutions with an online learning environment are basically based on individual development. Each site configuration of relevant hardware facilities generally allows only a certain number of users to access them when a large number of users can visit the site simultaneously. The system can easily collapse, and with the increasing number of learning resources, storage space appears slightly stretched. Although some of the wealthy units upgrade hardware facilities to cope with the aforementioned situation, these hardware resources are wasted in most cases. In today's knowledge-based society where resource sharing is crucial, the advantage of real-time contact is highly evident. Exploring and solving problems in online learning development for the dissemination of educational information are essential. Therefore, this study builds a new online learning system based on the popular Google cloud computing platform. Experiments show that the proposed platform can effectively meet the learning needs of teachers and students, and thus, is expected to receive their support.

\section{State of Art}

After online learning was introduced to the country, distance education in China in the form of radio and television has become the basis for the gradual development of network education as the main form of modern distance education. Tsinghua University, Zhejiang University, Hunan University, and Beijing University of Posts and Telecommunications, which are four ordinary higher-learning institutions, conducted pilot studies on the institution of modern distance education through a large class of professionals. After over 10 years of development, the Ministry of Education of China has approved small network education pilot schools, with tens of thousands of smallscale learning cores and the number of students reaching several millions. In addition, internal staff's training methods for online learning applications have increased rapidly, with most leading enterprises in the country establishing their own online learning network environment. With the progress of society, traditional online learning platforms demonstrate an uneven distribution of information resources and the information island phenomenon is evident. Online learning could be achieved by using the current dating platform [2]. However, the shortcomings of this platform are apparent, given that dealing with the relationship among suppliers is difficult. In addition, online streaming media technology to achieve online learning was proposed [3]. Streaming media technology resources demonstrate sharing advantages, and thus, they can provide considerable benefits for online learning. However, these resources require a large download memory, which is a huge flaw because it results in network delay. Scholars are continuously developing new methods. For example, someone suggested building through the Hadoop cluster to achieve online learning [4]. Howev- 
er, the cluster builds more than one machine, and the requirements of each machine are relatively high; therefore, construction cost can be prohibitive.

Through the "Google 101" program, Google, in cooperation with China Taiwan University, Taiwan Jiaotong University, and Tsinghua University in Mainland China, implemented a cloud computing project in early 2008. Other industry giants were closely following the pace of cloud computing and were soon launching their own cloud computing education projects, such as IBM's "Blue Cloud" program and Amazon's "Cloud Computing Open-end Funds." The "Hangzhou Normal UniversityMicrosoft IT College" was also established. In summary, these domestic and foreign projects for training cloud computing-related skills lay a solid foundation for both business and education, build a bridge to establish cooperative relationships, and further promote cloud computing in the field of education. Although cloud computing started relatively late in China, its market prospects are highly optimistic based on current development trends. The China Education Technology Association was held in Shanghai on May 16-17, 2009.As the first "cloud learning assistant teaching advanced training course" held in the country, it aims to help qualified schools master the theory and technology of cloud computing teaching to promote basic education and teaching reforms in China, thereby improving the quality of teaching in the country.

If the cloud computing research project of Google and Tsinghua University opened the door to cloud computing in China, then the advanced training in cloud-based teaching sowed the seeds of cloud computing in the country. After this training, primary and secondary schools in the country gradually began to practice cloud-based teaching [5]. Universities and companies began to establish cloud computing cooperation research projects for college teachers and business employees, and then for primary and secondary school teachers. This situation fully illustrates that China has adopted cloud technology as an educational application to promote the development of curriculum reform in the country and to challenge traditional education. However, although cloud technology was involved in the field of education in both the original Google 101 program or the cloud-assisted teaching training in China, it was mostly limited to a single-level teaching application. Therefore, in the latter part of promoting cloud computing application in education, new application areas should be explored to take full advantage of the high efficiency of cloud computing [6]. Unlike other new technologies, cloud computing, with its low threshold advantage, requires the support of schools. With the cloud computing in the field of education in the application of the gradual in-depth, and its educational support function has gradually been recognized, for people's attention. An in-depth analysis of the cloud education support function will help us develop other educational applications and fully demonstrate the educational support function of cloud computing. 


\section{$3 \quad$ Methodology}

\subsection{Concept and characteristics of online learning}

Online learning is based on network technology and multimedia technology, which utilize the Internet for learning and teaching activities. It makes full use of modern information technology to provide a convenient and efficient communication mechanism and a resource-rich learning environment for information age learners, and consequently, builds a new learning model [7]. This learning mode changes the teaching mode in which teachers 'unified teaching in all the traditional teaching and uses the characteristics of Internet resource sharing to address the drawbacks of limited knowledge sources in traditional teaching. Online learning has three characteristics; that is, it is networked, personalized, and tracked.

- Networked: Through network connection, learning resources can be updated in a timely manner to ensure the consistency of knowledge.

- Personalized: Students use their own networked equipment to learn according to their own time and learning needs and to establish their own learning progress and learning content.

- Tracked: The online learning platform can record all the learning activities of students and provide the basis for assessing intelligent learning and learning outcomes.

The difference of web-based online learning from the traditional classroom learning model is evident mainly in the following aspects. Online learning makes independent learning a reality. Learners are no longer constricted by time, place, and learning resources. As long as a computer is connected to the Internet, students can choose their learning time and learning content, and thus, self-learning becomes inevitable [8]. Online learning makes interactive cooperative learning a reality. Through the Internet, learners cannot only browse courseware, download information, and submit homework, but they can also use email, chat tools, forums, and other interactive means to communicate with their teachers and other students. They can discuss knowledge learned through interactive exchanges, which helps motivate students to learn $[9,10]$. Online learning makes individual learning a reality. In the traditional classroom teaching, many learners use common teaching materials and exhibit similar learning progress. By contrast, in the network-based online learning model, learners can study according to their own learning characteristics. They are free to choose the appropriate learning content and learn following their own approach and speed. This feature highlights the characteristics of an individual. Figure 1 shows the framework of the traditional online learning platform. 


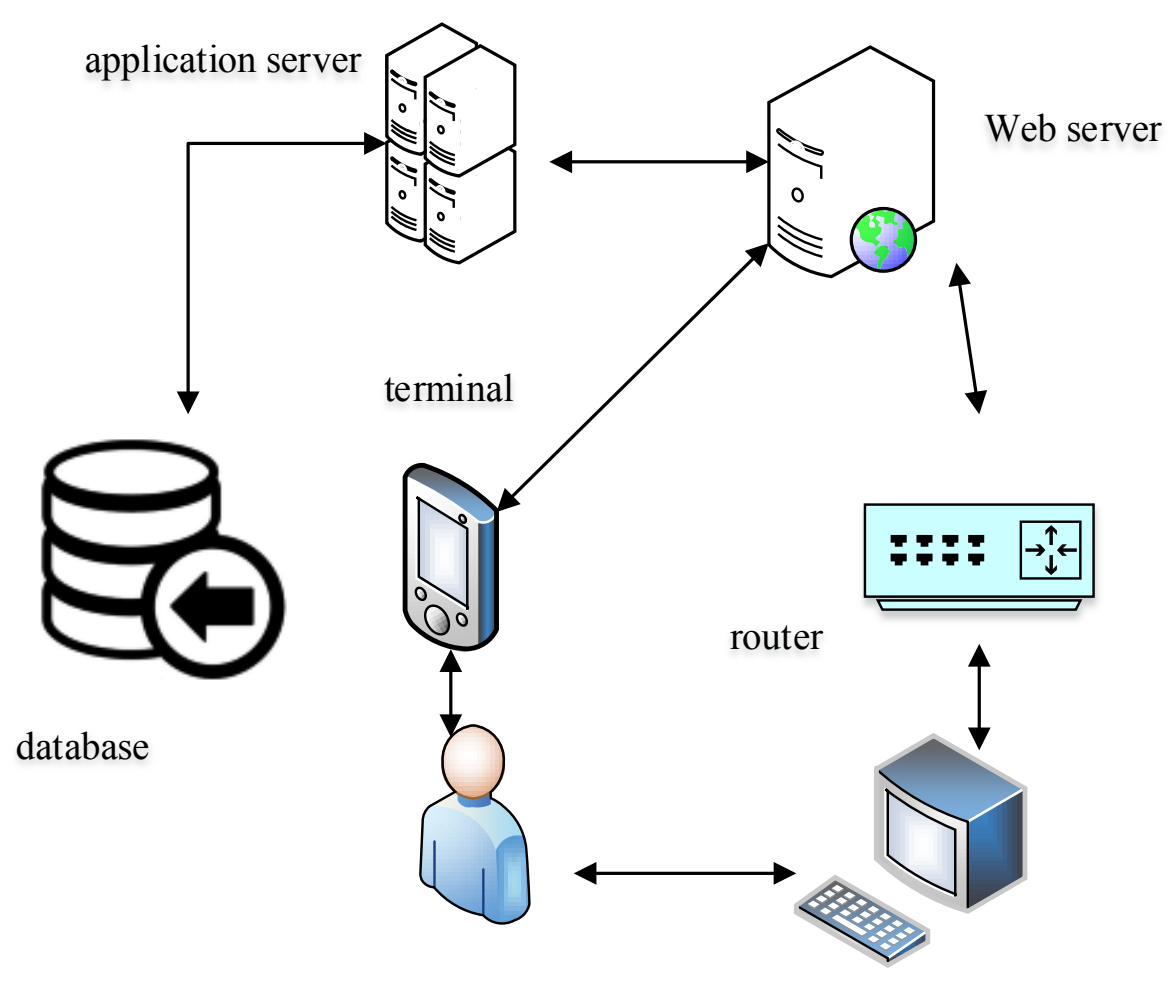

Fig. 1. Traditional online learning platform

\subsection{Cloud computing}

Cloud computing is a flexible and easy-to-access model. It is a configurable shared computing resource pool that can be minimized for network, server, and storage applications and services. Cloud computing can be quickly provisioned and released. The concept of cloud computing varies with different technologies, risks, attributes, and characteristics due to various uses and times. To business providers and the market, cloud computing technology has brought considerable economic benefits as it gradually develops. Cloud can exert large-scale impact on the industrial business model. The cloud computing architecture provided by the National Technology and Standards Bureau of Information Technology is as shown in Figure 2.

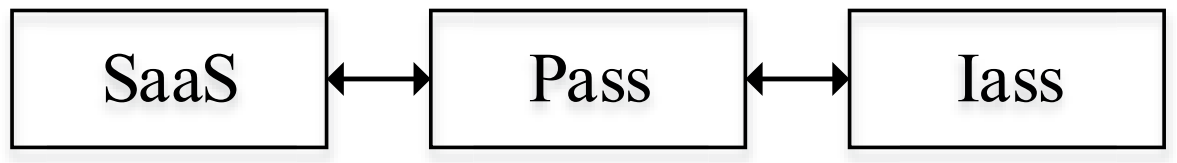

Fig. 2. Cloud computing architecture 
The five basic characteristics of cloud computing are summarized as follows: resource on-demand self-service; anytime and anywhere network access; distributed independent shared resource pool; fast, convenient, and flexible integration and release of resources; and measurable service effectiveness [11, 12]. Network users do not need to interact directly with the network service provider to ask for help with regard to server demand, network storage, and other computing resources. A variety of network clients can be available anytime, anywhere through a standard application and interface that can be accessed by network users who need to know the division of the resource pool or storage; computing, processing, storage, and network bandwidth; virtual machine number; and other data. Or master the resource pool of specific technology can be applied to them operation, the network provider to the pool of computing resources in the form of tenants could provide services only by the needs of the network consumers need to dynamically allocate or release different physical resources, virtual resources. Network users can rapidly, conveniently, and flexibly integrate and release resources and quantify them at all times. They can detect and control the use of resources, and a transparent reporting of suppliers and users is feasible to control and optimize the resources of a cloud system in a measurable manner. The cloud computing deployment model is shown in Figure 3.

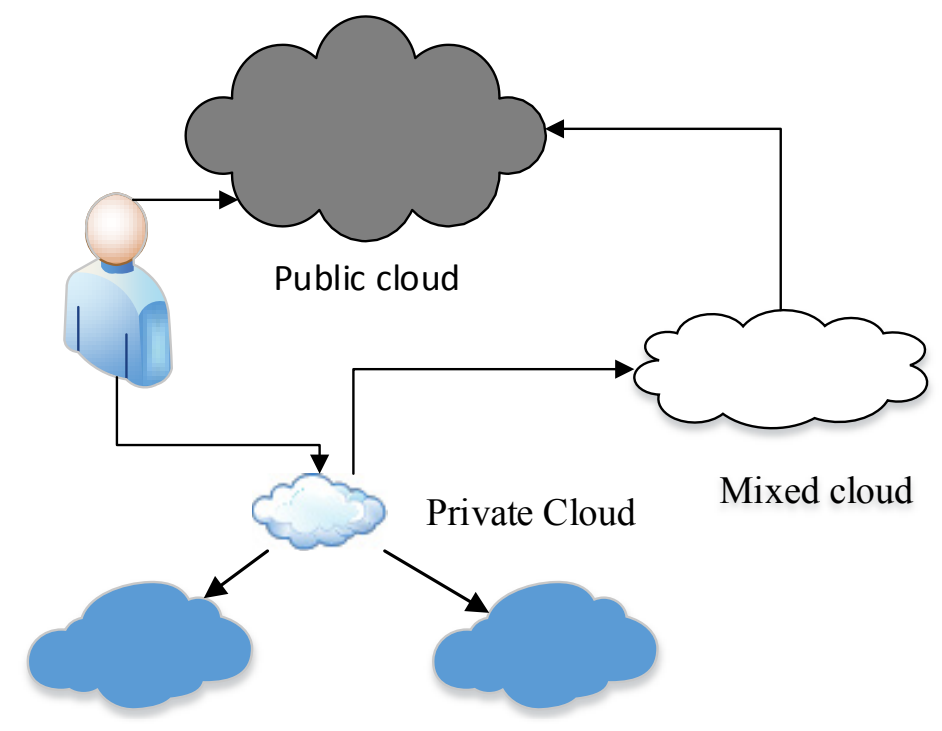

\section{Local personal hosting service}

Local third-party platform hosting

Fig. 3. Cloud computing deployment model

The three levels of cloud computing services are infrastructure, software, and platform cloud services. The corresponding cloud computing delivery model is summarized as follows. The infrastructure cloud service delivery model represents the network, computing, and storage resources. The platform cloud service delivery model represents the building and deployment of cloud services in a development environ- 
ment. The software cloud service delivery model represents the individual users and the software application infrastructure. Cloud service is the provision of storage resources, network resources, and other computing resources to network users [13]. Network users deploy on-demand and run their operating systems and other applications. They simply deploy operating systems on the infrastructure and select network elements, such as storage, firewall, and load balancing devices, without having to manage and control the facilities in the cloud. Infrastructure cloud services refer to the utilization of technical component services. Google and Amazon provide virtual machines and other infrastructure services only if the network is able to access a group of actual available machines, thereby saving time and costs required in an actual machine installation deployment process. Through a cloud of the service cluster or a cloud of a separate server cloud service virtual machine network, users can increase available resources on demand or release unused resources and calculate the use of time, bandwidth, and storage. Network users can use infrastructure cloud services to deploy the required applications to the cloud and build their own private cloud platform, which will not only reduce the cost of technical facilities but will also run existing applications on the cloud. Figure 4 shows how cloud computing is implemented.

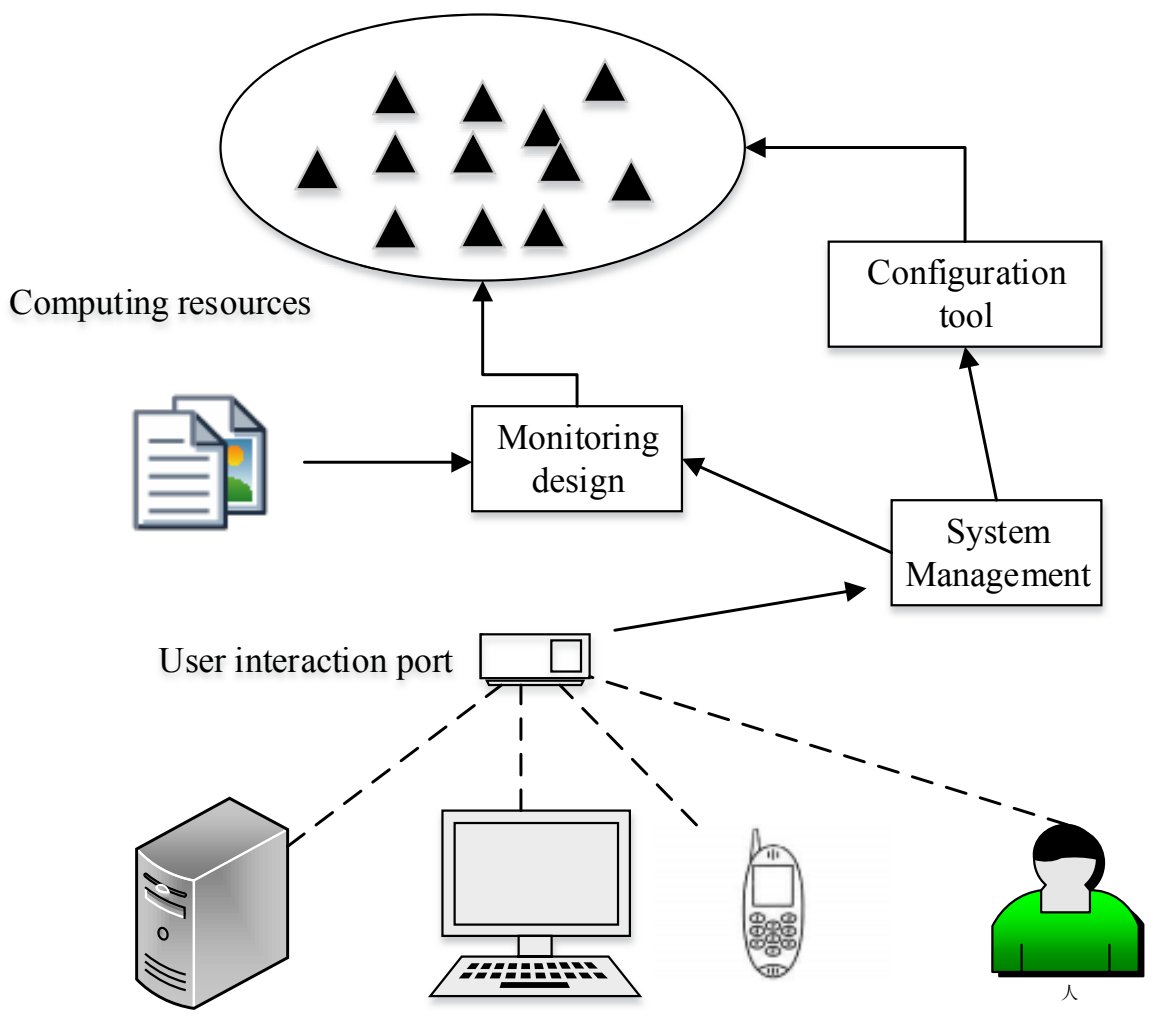

Fig. 4. Implementation mechanism of cloud computing 


\subsection{Design of online learning system based on Google cloud computing}

The cloud platform, which has the capability to store large amounts of data and integrate a considerable amount of learning resources on the cloud, does not only provide storage, server, and network equipment, as well as infrastructure services, but also strong computing power, massive spatial data storage capacity and communication resources, and can even solve the problem of resource independence. The cloud platform has been set up in an online learning system to enable users to access all the learning materials stored in the cloud and practice online learning through a unified interface. The data of a unit can also be stored, collected, and managed through the cloud platform. Cloud service resources by specialized cloud platform providers are responsible for the operation and management of data; hence, the application layer of the education platform will no longer worry about server security issues. A traditional online learning system may consist of a few servers. By contrast, a cloud platform server cluster is composed of millions of servers. A traditional online learning system is supported only by a small number of terminal servers compared with a cloud platform, which has a more robust network.

An online learning platform requires learning and computing resources, storage and application systems, and a specific user interface to run a perfect operation. A detailed illustration of an online cloud platform to set up relevant rules and techniques is shown in Figure 5. In a cloud computing system, users and clients act as consumers, whereas the data layer, application layer, infrastructure layer, and management platform layer play the roles of supporters and service providers. Students, teachers, and system administrators are the three types of online learning platform. The client assumes the media interaction function, which provides users with different interfaces and functions, as well as displays system content to the user according to different types of user equipment. The management platform layer can provide the operating environment for the application layer in an online learning system, and it plays a central role in the entire cloud platform architecture. A cloud computing platform corresponds to a three-tier service platform. The service layer, or the PaaS system, is a cloud computing system based on Google on a third-party application platform. The data layer is the central layer of the entire framework; it manages all types of data storage and is the foundation of the learning platform. The infrastructure layer determines the service capacity and the scale of the cloud platform. The corresponding infrastructure in the cloud computing service layer is the service, which is the basic layer of the entire structure.

\subsection{System function module design and system components of the effect display}

A detailed analysis of the demands of an online learning system shows that such system is divided into an interactive communication module, a learning resource management module, a user management module, and a test module. 


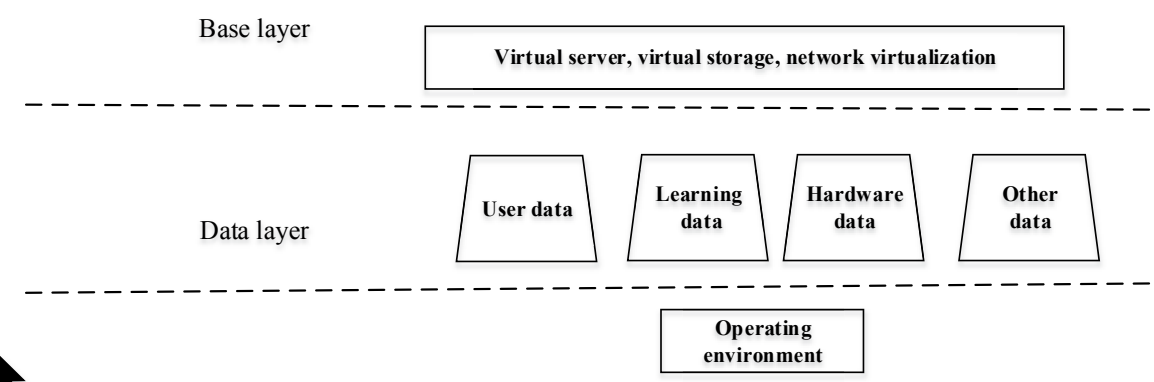

Management layer
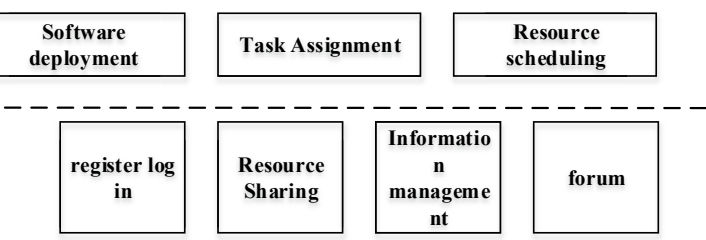

Application layer

Online learning system

Client

Terminal Equipment

User layer

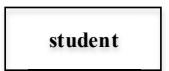

teacher

administrator

Fig. 5. Online learning platform structure based on Google cloud computing

- User management module: This module mainly comprises the authorization, registration, and audit management settings of the user to ensure that users participating in the learning process will have legitimate access and operational behavior.

- Public information module: This module is a function module designed for information, such as website information, news information, and announcements. A website profile contains dynamic messages and provides service information, which presents a brief description of the basics of learning the website. The site offers various permissions to different users. For example, students can only view, teachers can publish and delete messages, and the administrator can manage and classify information categories.

- Resource management module: This module generally includes curriculum management and learning resource management. For example, if you wish to accommodate additional content, then you can make two small systems: a curriculum management system to enable the addition and deletion of curriculum courseware and a learning resource management system to add and manage course-related learning materials. 
- Test module: This module provides the functions of adding, deleting, and modifying test papers and determining scores. In this manner, the majority of the students will aim to fill in the gap, whereas the teachers will aim to understand the actual level of the required learning of the students.

- Interactive communication module: This module allows students and teachers to communicate via e-mail, forums, and instant messaging. These means of interaction are timely and convenient.

The picture shown in Figure 6 presents an example of the interaction between teachers and students in a test system.

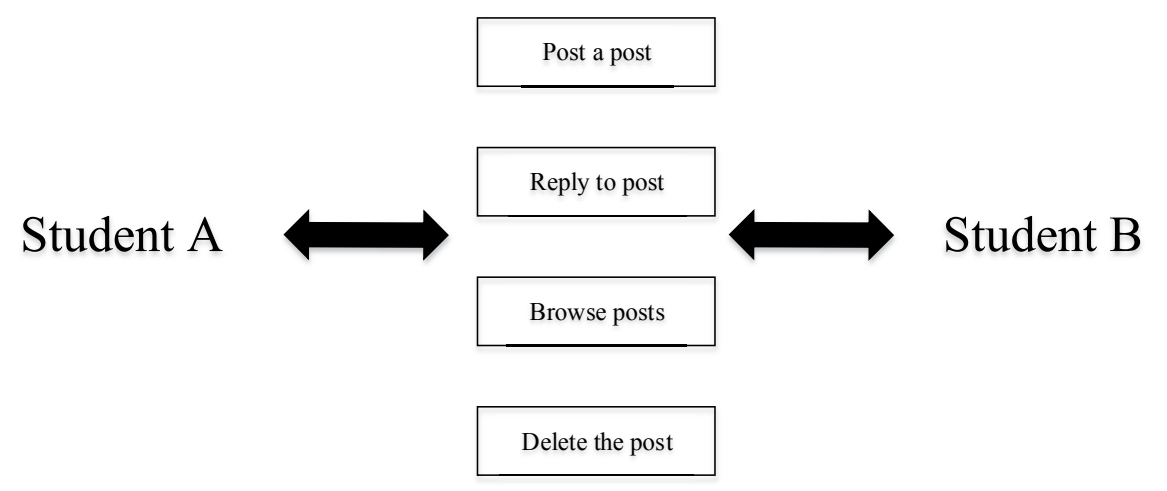

Fig. 6. Analysis of the interaction between students

\section{Result Analysis and Discussion}

With the continuous development of cloud computing, many enterprises have launched a variety of cloud computing products and services. Cloud computing is now widely recognized, and its core technologies include the distributed file system, distributed computing programming model, and distributed data storage system. They are also behind the platform will be a strong support.

\subsection{Distributed file system}

A document system, which is a large distributed file system (Figure 7), is mainly used to deal with the rapid growth of cloud computing data. It has the same objectives as the traditional distributed file system, such as scalability, availability, and reliability. However, its design principles and implementation ideas are based on a unique technical environment and application requirements, in which large data storage and processing are performed [13]. The system nodes are divided into three categories: client, main server, and data block server. A client is provided to the application of the access interface to the form of library files available, the application can directly call these library functions. The main server is a distributed file management system node. It is mainly responsible for managing the entire file system and saving system 
metadata. The data block server is responsible for specific storage. Data are stored in the form of files, and the number of clients determines the size of the distributed file system.

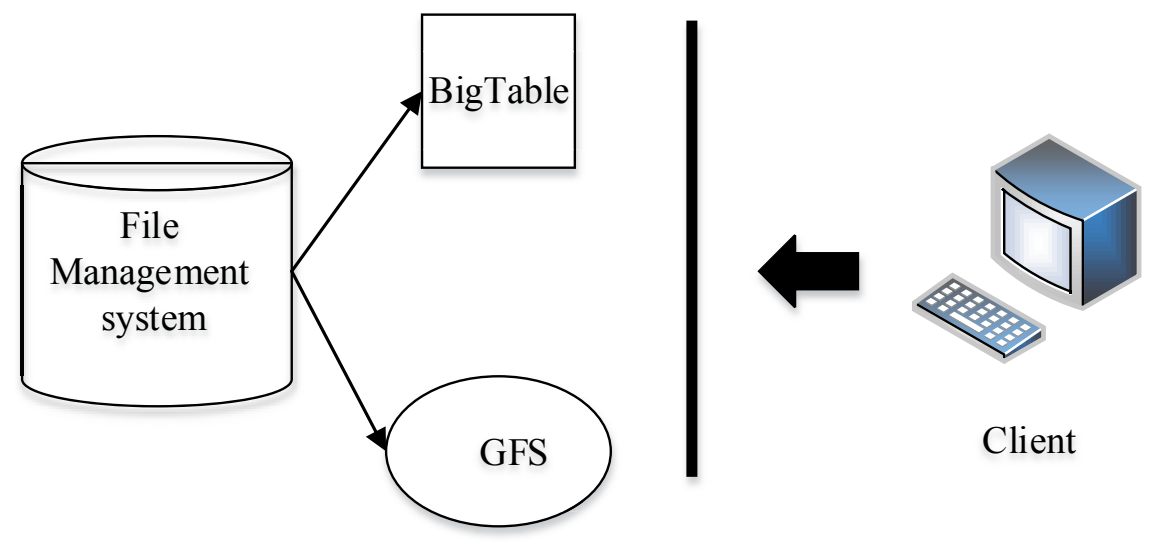

Fig. 7. File sharing system based on the Google cloud computing platform

When a client accesses the server, he/she first accesses the master server node, obtains data block server information to interact with it, and then directly accesses complete data. Thus, a client directly accesses the main server to transfer data information, and no datum is accessed between data block interaction. When interactive data are divided into multiple data block distributed storage, a client can simultaneously access multiple data block servers. This design does not only reduce the load of the data block server, but also the parallel processing of the input and output of the entire system, thereby improving overall performance.

A file management system uses the central server model to manage the entire file system, thereby significantly improving system scalability. All the operations initiated by a client should be implemented first, and the master server should control the situation within the entire system. The addition of a new data block server should only be registered with the main server, and updating the information to inform each server is unnecessary. Consequently, the expansion and contraction of the storage block becomes easy [14]. A file management system applies a copy of the method to achieve fault tolerance. Each datum has at least three different locations on the machine. Operating system problems, network interruptions, or sudden power failures interrupt the hard disk data storage process. The fault detection and automatic recovery features of the file management system will enable recovery of damaged documents, and thus, reliability is highly guaranteed.

\subsection{Google app engine (GAE)}

The GAE cloud computing platform for software developers, which exhibits vast computing power and storage space, is hosted directly by Google. It allows users to deploy and run applications directly on a powerful infrastructure. 


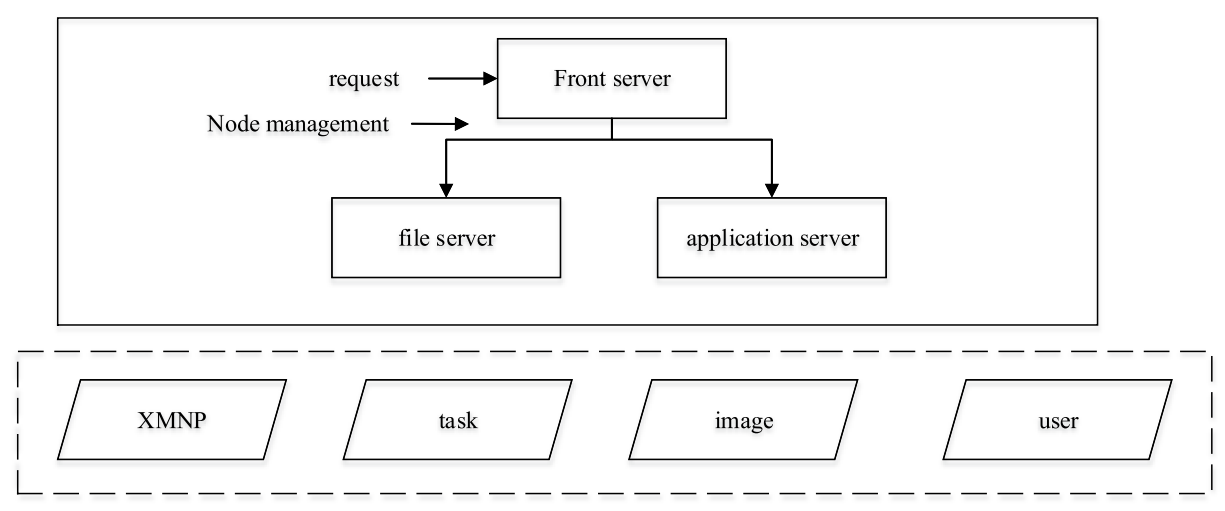

Fig. 8. GAE structure

The GAE architecture, which is shown in Figure 8, can be divided into three main parts: the front end, the data storage area, and the service group. The front part includes four modules: the front-end server, static file server, application server, and application management node. The front-end server can be considered a load balancer or a proxy server. It is mainly responsible for load balancing and forwarding the request to the application server or the static file server. The static file server is similar to the content distribution network; it is used to store and send pages attached to the static files, such as pictures, style sheets, and scripts. The application server is mainly used to deal with a request sent by an end user and in accordance with the request to call data or external services, generate a response, and return. The use of a programming language is divided into different versions of the application server. The application management node is responsible for monitoring the application load and the scheduling applications. The monitoring and scheduling results will be sent to the front-end server, and then according to the load situation on the request to achieve the application of scalability.

Data storage area: The main data store of GAE is Datastore. It is a distributed storage service based on Google Bigtable technology that automates scalability issues, such as creating backups, clustering, and load balancing data, thereby infinitely expanding data storage capacity. Datastore in Bigtable is based on the realization of the data storage model and query function.

The basic unit of data storage is an entity. One entity is equivalent to a record in the relational database table. Multiple instances of the same entity form a family, which is similar to multiple records in a relational database that form a table. A comparison between Datastore and the traditional relational database is provided in Table 1. As shown in the table, Datastore is not optimal in terms of response speed and write data, among others; however, it can support massive data storage and query with good scalability. Web applications are mainly used to read the main, and will continue to produce a massive amount of data, and thus, Datastore is highly suitable for webtype application data storage. 
Table 1. Comparison between Datastore and traditional database

\begin{tabular}{|l|l|l|}
\hline \multicolumn{1}{|c|}{ Content } & \multicolumn{1}{c|}{ Datastore } & \multicolumn{1}{c|}{ Traditional Database } \\
\hline SQL support & Support part of the query & Complete support \\
\hline Main structure & Level & Relationship \\
\hline Thing & Support is performed within an entity group & Fully supported \\
\hline Execution speed & Less than $200 \mathrm{~ms}$ & Less than $100 \mathrm{~ms}$ \\
\hline Extensibility & Simple & Difficult \\
\hline
\end{tabular}

\subsection{Teaching effects}

This study validates the feasibility and effectiveness of an open online learning platform based on Google cloud computing. In classes A and B, two classes were implemented in this study teaching methods and traditional teaching methods to carry out $\mathrm{C}$ program curriculum design. After completing the courses for these two teaching methods, the learning situations of the students are evaluated, and the survey results are presented in Figure 9.

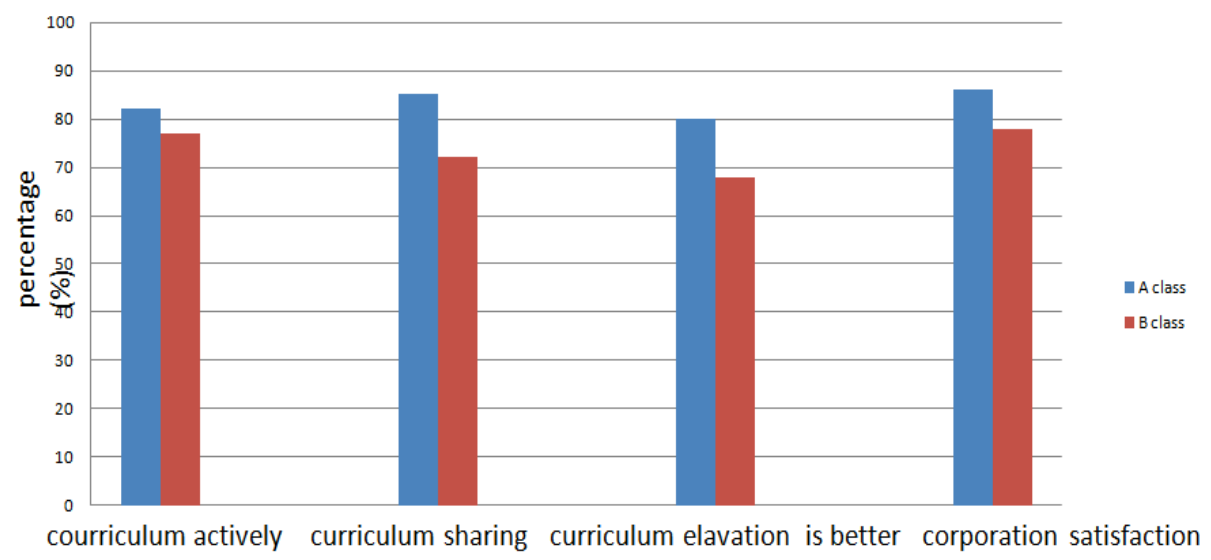

Fig. 9. Results of the teaching effect

As shown in figure 9, Class A uses the teaching method proposed in this study and performs better than Class B, which uses the traditional teaching method. The students in Class A are more interested in $\mathrm{C}$ language programming, classroom learning atmosphere is more active, teacher information resource sharing is better. Curriculum design evaluation is also better in Class A than in Class B. After graduation, Class A students are more satisfied with their performance, thereby indicating that the teaching method is feasible and has a positive effect on teaching. 


\section{Conclusions}

In view of the problems in the current online learning system, namely, slow resource sharing and limited number of resources, we first analyzed the current online learning situation in China. Research on the cloud computing platform to build a possible online learning system was becoming popular. Finally, we built our own cloud computing online learning platform based on the Google cloud platform. After design implementation and testing, the following conclusions are obtained:

1. The use of a cloud computing platform can provide a good online learning interactive system in real time.

2. The system can provide considerable shared resource storage support.

3. Research on cloud computing for online learning system design has great prospects.

\section{Acknowledgment}

This research is supported by Soil Moisture Monitoring based on the Google cloud service platform (No. 15A120011).

\section{$7 \quad$ References}

[1] Sakellari G, Loukas G. A survey of mathematical models, simulation approaches and testbeds used for research in cloud computing. Simulation Modelling Practice and Theory, 2013, vol.39, pp.92-103. https://doi.org/10.1016/j.simpat.2013.04.002

[2] Liyanagunawardena T R, Adams A, Williams S A. MOOCs: A systematic study of the published literature 2008-2012. The International Review of Research in Open and Distributed Learning, 2013, vol.14(3), pp.202-227. https://doi.org/10.19173/irrodl.v14i3.1455

[3] Hashizume K, Rosado D G, Fernández-Medina E, et al. An analysis of security issues for cloud computing. Journal of Internet Services and Applications, 2013,vol.4(1), pp.5. https://doi.org/10.1186/1869-0238-4-5

[4] Dinh H T, Lee C, Niyato D, et al. A survey of mobile cloud computing: architecture, applications, and approaches. Wireless communications and mobile computing, 2013, vol.13(18), pp.1587-1611. https://doi.org/10.1002/wcm.1203

[5] Fernández A, Peralta D, Benítez J M, et al. E-learning and educational data mining in cloud computing: an overview . International Journal of Learning Technology, 2014, vol. 9(1), pp.25-52. https://doi.org/10.1504/IJLT.2014.062447

[6] Abolfazli S, Sanaei Z, Ahmed E, et al. Cloud-based augmentation for mobile devices: motivation, taxonomies, and open challenges. IEEE Communications Surveys \& Tutorials, 2014, vol.16(1), pp.337-368. https://doi.org/10.1109/SURV.2013.070813.00285

[7] González-Martínez J A, Bote-Lorenzo M L, Gómez-Sánchez E, et al. Cloud computing and education: A state-of-the-art survey. Computers \& Education, 2015, vol.80, pp.132151. https://doi.org/10.1016/j.compedu.2014.08.017

[8] Lim N, Grönlund Å, Andersson A. Cloud computing: The beliefs and perceptions of Swedish school principals. Computers \& Education, 2015, vol.84, pp.90-100. https://doi.org/10.1016/j.compedu.2015.01.009 
Paper-Exploration of an Open Online Learning Platform Based on Google Cloud Computing

[9] Abolfazli S, Sanaei Z, Tabassi A, et al. Cloud adoption in Malaysia: Trends, opportunities, and challenges. IEEE Cloud Computing, 2015, vol.2(1), pp.60-68. https://doi.org/10.1109/ MCC.2015.1

[10] Hashem I A T, Yaqoob I, Anuar N B, et al. The rise of "big data" on cloud computing: Review and open research issues. Information Systems, 2015, vol.47, pp.98-115. https://doi.org/10.1016/j.is.2014.07.006

[11] Nguyen T D, Nguyen T M, Pham Q T, et al. Acceptance and use of e-learning based on cloud computing: the role of consumer innovativeness.International Conference on Computational Science and Its Applications. Springer International Publishing, 2014, pp.59174. https://doi.org/10.1007/978-3-319-09156-3 12

[12] Mojica I J, Adams B, Nagappan M, et al. A large-scale empirical study on software reuse in mobile apps . IEEE software, 2014, vol.31(2), pp.78-86. https://doi.org/10.1109/ MS.2013.142

[13] Sanaei Z, Abolfazli S, Gani A, et al. Heterogeneity in mobile cloud computing: taxonomy and open challenges. IEEE Communications Surveys \& Tutorials, 2014, vol.16 (1), pp.369-392. https://doi.org/10.1109/SURV.2013.050113.00090

\section{Authors}

Kai Zhao is with Information Engineering College,North China University of Water Resources and Electric Power, Zhengzhou, China (zhaozhangk@126.com).

Qingxue Yang is with Information Engineering College, North China University of Water Resources and Electric Power, Zhengzhou, China

Xinming Ma is with HenNan Agricultural University, Zhengzhou, China

Article submitted 03 June 2017. Published as resubmitted by the authors 07 July 2017. 